\title{
Deep Learning-Based Decision Region for MIMO Detection
}

\author{
Termeh Faghani*, Arman Shojaeifard ${ }^{\dagger}$, Kai-Kit Wong ${ }^{\S}$, and A. Hamid Aghvami* \\ *King's College London, London WC2R 2LS, UK \\ †T Labs, Adastral Park, Ipswich IP5 3RE, UK \\ $\S$ University College London, London WC1E 7JE, UK \\ *termeh.faghani@kcl.ac.uk †arman.shojaeifard@bt.com ${ }^{\S}$ kai-kit.wong@ucl.ac.uk *hamid.aghvami@kcl.ac.uk
}

\begin{abstract}
In this work, a deep learning-based symbol detection method is developed for multi-user multipleinput multiple-output (MIMO) systems. We demonstrate that the linear threshold-based detection methods, which were designed for AWGN channels, are suboptimal in the context of MIMO fading channels. Furthermore, we propose a MIMO detection framework which replaces the linear thresholds with decision boundaries trained with neural network (NN) classifiers. The symbol error rate (SER) performance of the proposed detection model is compared against conventional methods under state-of-theart system parameters. Here, we report to up to a $2 \mathrm{~dB}$ gain in SER performance using the proposed NN classifiers, allowing for exploiting higher-order modulation schemes, or transmitting with reduced power. The underlying gain in performance may be further enhanced from improvements to the NN architecture and hyper-parameter optimization.
\end{abstract}

\section{INTRODUCTION}

An end-to-end communications system consists of many blocks which are typically optimized separately either based on tractable channel models or through real-world measurements. With networks becoming increasingly more complex, it is no longer feasible to develop accurate tractable models, and results from field trials may not be adequate for capturing largescale deployments. These trends have opened the door for the application of machine learning in communications system design and optimization [1]. In [2], the authors discussed the different data-driven and modeldriven deep learning-based approaches. Therein, while an abundant number of labeled data was used to train each communications block, the data-driven approach did not outperform the fundamental information theoretic algorithms. On the other hand, the model-driven deep learning-based approach utilizes both machine learning algorithms and communications theory models.

The work in [3] utilized auto-encoders for training the transmitter and receiver under a given channel model, such to minimize the error performance. In [4], autoencoders were adopted for designing a multiple-input multiple-output (MIMO) system, wherein, constellations for $2 \times 2$ channels were learned. In [5], deep learning was applied to the maximum-likelihood (ML) detection problem for MIMO systems. The symbol error rate (SER) of their proposed "DetNet" versus conventional receivers was studied. Given machine learning models are trained offline, there may exist a large mismatch between the learned model and the actual online system. This trend was studied in [6], where a two-phase training strategy based on transfer learning was proposed. Specifically, in the first phase, the auto-encoder was trained on the stochastic channel model, and then in the second phase, they finetuned the receiver by training on the real channel. Their neutral network (NN)-based system performed $1 \mathrm{~dB}$ worse than the baseline model, however, improvements may be achievable via hyper-parameter tuning and adjustments to the NN architecture.

Existing MIMO detection methods using deep learning feed the received signal and the processed channel information as separate inputs of the learning method. In this paper, we argue that this approach fundamentally downgrades performance, as the NN cannot optimally form an estimate of the signal with linear channel processing. In conjunction with this notion, we illustrate that the linear detection thresholds are a sub-optimal solution for non-additive white Gaussian noise (AWGN) channels, and define how the optimal decision regions can be derived. Furthermore, we propose a novel deep learning-based detection model for multi-user MIMO systems, where NN classifiers are adopted to match the optimal non-linear decision boundaries. Our simulation results demonstrate the superior error performance of the proposed deep learning-based MIMO detection approach over conventional methods, allowing for achieving higher spectral or energy efficiencies.

Notation: The following notation is used in this paper. Bold upper-case and lower-case letters respectively correspond to matrices and column vectors; superscripts $\mathrm{T}, *$ and $\dagger$ respectively represent the transpose, conjugate-transpose, and pseudo-inverse operations; $p(x)$ is the probability of event $x ; \mathscr{C} \mathscr{N}(\mu, v)$ is the complex Gaussian distribution with mean $\mu$ and variance $v$. 


\section{System Model AND Assumptions}

Consider a multi-user MIMO system comprising single-antenna user equipments (UEs) communicating with a multi-antenna base station (BS). We use $N$ and $U$ to respectively denote the number of BS antennas and UEs - here, we assume $N \geq U$, hence, user scheduling mechanisms are not needed. An illustrative diagram of the uplink multi-user MIMO system under consideration is depicted in Fig. 1.

The received signal at the BS is a linear combination of the transmitted data symbols jointly affected by fading and noise. Let $y$ denote the baseband $N$-dimensional received signal vector at the BS. Mathematically,

$$
\boldsymbol{y}=\boldsymbol{H} \boldsymbol{x}+\boldsymbol{n}
$$

where $\boldsymbol{H}$ is the $N \times U$ fading channel matrix, $\boldsymbol{x}$ is the $U$ dimensional symbol vector, $\boldsymbol{n}$ is the $N$-dimensional noise vector. In this work, we utilise the independent Rayleigh block fading channel model, hence, the coefficients of $\boldsymbol{H}$ are drawn from $\mathscr{C} \mathscr{N}(0,1)$. Further, complex AWGN is assumed with elements of $\boldsymbol{n}$ distributed according to $\mathscr{C} \mathscr{N}(0,1)$. We consider channel state information (CSI) to be available at the BS through channel reciprocity or pilot training. Further, the transmitted symbols are constructed using the $M$-array quadrature amplitude modulation ( $M$-QAM) method. Note that $M=2$ and $M=4$ are special cases of $M$-QAM corresponding to binary phase-shift keying (BPSK) and Quadrature phaseshift keying (QPSK) schemes.

The received vector at the BS, $\boldsymbol{y}$, is constructed using the observations from all receive antennas. Considering a received vector $\boldsymbol{y}=\boldsymbol{v}$, the Bayes' theorem states [7]

$$
p_{\boldsymbol{x} \mid \boldsymbol{y}}(i \mid \boldsymbol{v}) p_{\boldsymbol{y}}(\boldsymbol{v})=p_{\boldsymbol{y} \mid \boldsymbol{x}}(\boldsymbol{v} \mid i) p_{\boldsymbol{x}}(i)
$$

where $p_{\boldsymbol{x}}(),. p_{\boldsymbol{x} \mid \boldsymbol{y}}(),. p_{\boldsymbol{y} \mid \boldsymbol{x}}($.$) denote the priori, posteriori,$ and channel transition probabilities, respectively. Hence, for $p_{y}(\boldsymbol{v}) \neq 0$,

$$
p_{\boldsymbol{x} \mid \boldsymbol{y}}(i \mid \boldsymbol{v})=\frac{p_{\boldsymbol{y} \mid \boldsymbol{x}}(\boldsymbol{v} \mid i) p_{\boldsymbol{x}}(i)}{p_{\boldsymbol{y}}(\boldsymbol{v})} .
$$

The optimal (BS) receiver is the maximum à posteriori (MAP) detector, defined as follows. Note that the denominator $p_{y}(\boldsymbol{v})$ is a constant, therefore, it is ignored when maximizing (3) over $i$.

Definition 1. The MAP detector selects the index $i$ to maximize the posteriori probability $p_{\boldsymbol{x} \mid \boldsymbol{y}}(i \mid \boldsymbol{v})$ given a received vector $\boldsymbol{y}=\boldsymbol{v}$. Assuming all input messages are equiprobable, the MAP rule can be described as

$$
\hat{m} \Rightarrow m_{i} \text { if } p_{\boldsymbol{y} \mid \boldsymbol{x}}(\boldsymbol{v} \mid i) p_{\boldsymbol{x}}(i) \geq p_{\boldsymbol{y} \mid \boldsymbol{x}}(\boldsymbol{v} \mid j) p_{\boldsymbol{x}}(j) \forall j \neq i
$$

For the MAP detector, after the estimation is formed, each and every value of $\boldsymbol{y}$ is mapped to one of the $M$

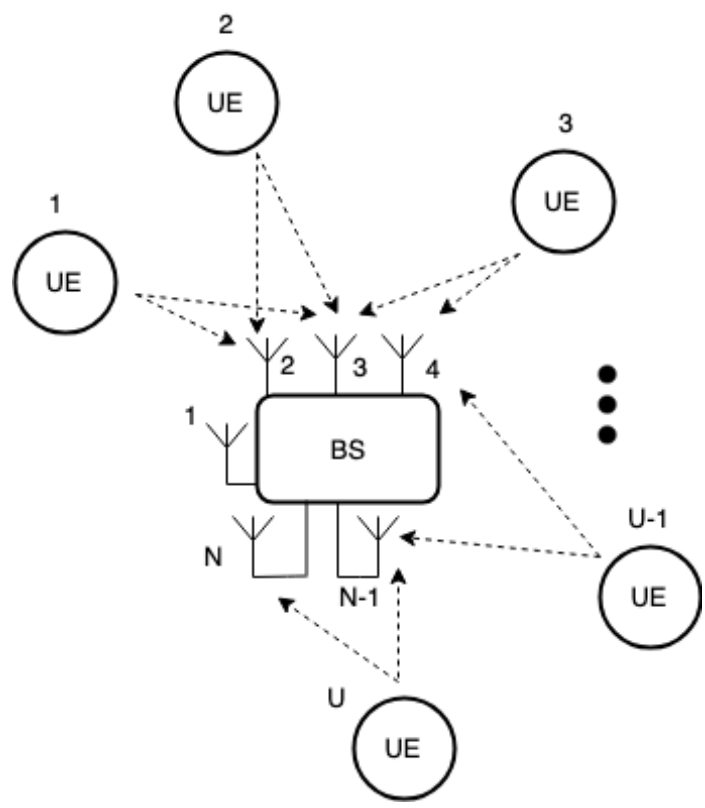

Fig. 1: Schematic diagram of the uplink multi-user MIMO system under consideration. The BS with $N$ antennas simultaneously receives signals from $U$ singleantenna UES.

points on the constellation diagram. Thus, the vector space of $y$ can be divided into $M$ different regions. The MAP decision region for M-QAM detection is mathematically defined as follows [7].

Definition 2. The decision region for $M-Q A M$, with messages $m_{i}, i \in\{0, \cdots, M-1\}$, using the MAP detector is given by

$$
D_{i} \triangleq\left\{\boldsymbol{v} \mid p_{\boldsymbol{y} \mid \boldsymbol{x}}(\boldsymbol{v} \mid i) p_{\boldsymbol{x}}(i) \geq p_{\boldsymbol{y} \mid \boldsymbol{x}}(\boldsymbol{v} \mid j) p_{\boldsymbol{x}}(j) \forall j \neq i\right\} .
$$

With uniformly distributed input messages, the above decision region reduces to

$$
D_{i} \triangleq\left\{\boldsymbol{v} \mid p_{\boldsymbol{y} \mid \boldsymbol{x}}(\boldsymbol{v} \mid i) \geq p_{\boldsymbol{y} \mid \boldsymbol{x}}(\boldsymbol{v} \mid j) \forall j \neq i\right\}
$$

For a basic communications system with AWGN channel, the decision regions can be readily derived given the channel transition probabilities are known. Specifically, minimizing the Euclidean distance from the constellation point, results in linear detection thresholds for AWGN channels [7]. There are however no closed-form expressions for the MIMO channel transmission probabilities under arbitrary signal-to-noise ratios (SNRs).

Consider the MAP detector for the multi-user MIMO system in (1)

$$
\hat{\boldsymbol{x}}(y)=\underset{\boldsymbol{x} \in \boldsymbol{\theta}}{\arg \min }\|\boldsymbol{y}-\boldsymbol{H} \boldsymbol{x}\|^{2}
$$


where $\hat{\boldsymbol{x}}$ is the $U$-dimensional estimated received symbol vector, and $\theta$ is used to denote the set of $M$ constellation points [8]. Solving (7) is not feasible in practice, since it involves an exhaustive search of $O\left(M^{U}\right)$ for all possible values of $\boldsymbol{x}$. As a result, sub-optimal detectors are commonly used in practice, such as the linear zeroforcing $(\mathrm{ZF})$ detector.

The estimated symbol, $\boldsymbol{x}_{\text {est }}$, from the output of the linear ZF detector, can be written as

$$
\boldsymbol{x}_{\mathrm{est}}=\boldsymbol{H}^{\dagger} \boldsymbol{y}
$$

where $\boldsymbol{H}^{\dagger}=\left(\boldsymbol{H}^{*} \boldsymbol{H}\right)^{-1} \boldsymbol{H}^{*}$ is the puesdo-inverse of the MIMO fading channel. Hence,

$$
\boldsymbol{x}_{\mathrm{est}}=\boldsymbol{H}^{\dagger} \boldsymbol{H} \boldsymbol{x}+\underbrace{\boldsymbol{H}^{\dagger} \boldsymbol{n}}_{\text {error }} .
$$

The detector hence attempts to map $x_{\text {est }}$ to $x$ based on the decision regions. However, the error term in (9) no longer follows a Gaussian distribution with known statistics, and no closed-form expression for this exists to date. Although the error distribution dictates the optimal decision regions for detection, conventional methods use sub-optimal linear detection thresholds, which were designed for AWGN channels. Linear thresholds perform poorly especially in low SNR, and for modulations of higher order, where the constellation points are closer.

\section{DEEP LEARNING-BASED DETECTION}

In this work, we propose a novel deep learning-based detection method, where the received signal is mapped to the corresponding constellation points based on nonlinear thresholds that are set by NN classifiers. In what follows, we formulate the proposed deep learning-based multi-user MIMO detector.

Proposition 1. Let $x[j]$ and $\hat{x}[j]$ respectively denote the transmitted and predicted symbols with respect to the transmit antenna (UE) $j$, where $j \in\{1, \cdots, U\}$. We denote the Hamming loss with $\mathscr{L}$, which captures the proportion of mismatch between the transmitted and predicted symbols. The MAP detector can be defined as

$\hat{h}_{M}(\boldsymbol{y}, \boldsymbol{H})=\underset{\hat{\boldsymbol{x}}}{\arg \max } \sum_{\boldsymbol{x}} \frac{p\left(\boldsymbol{x} \mid \boldsymbol{x}_{\text {est }}\right)}{U}\left(1-\sum_{j=1}^{U} \mathscr{L}(x[j], \hat{x}[j])\right)$.

In order to tackle (10), we propose a multi-label classifier using an artificial $\mathrm{NN}$ architecture. NN takes the central assumption that $p\left(\boldsymbol{x} \mid \boldsymbol{x}_{\text {est }}\right)$ can be approximated as a non-linear function applied to the linear combination of input features.

Remark 1. Note that the estimation loss can be defined for AWGN channels based on the Euclidean distance between the transmitted and received symbols, whereas for fading channels, it should be defined based on the Hamming distance.

Remark 2. Note that in (10), we utilize $\boldsymbol{x}_{\text {est }}$, which exploits both $\boldsymbol{y}$ and $\boldsymbol{H}$ to design a detector for the model in (9). However, some of the existing works in the literature formulate the detector based on (1). The latter approach does not lead to promising results given using only $\boldsymbol{y}$, instead of $\boldsymbol{x}_{\text {est }}$, means performing the detection with no channel knowledge.

Here, we have a multi-label multi-class classification problem. The problem is multi-label since we have multiple single antenna users, which the detector has to detect the transmitted symbols for, and it is also multi-class due to the fact that there is muti-class of symbols to detect depending on the constellation. The training data set consists of pairs of an input vector, which in our model is the received signal after forming the estimation, and the corresponding output vector, which consists of transmitted symbols. We use the binary relevance method [9] for the multi-label classification task, meaning the target labels for the classification are independent of each other. This assumption is in line with the multi-user MIMO system model, where UEs transmit symbols independently of each other to the BS. The symbols are generated randomly with uniform distribution with respect to the desired constellation. Then, they are passed through MIMO fading channel realizations for a wide range of SNRs. Next, at the receiver side, the received signals are fed to the estimator. The output of the estimator is used as the input training vector for the $\mathrm{NN}$ and the target values are the corresponding transmitted symbols.

Generally, applying machine learning algorithms to communications systems is challenging given the training data is complex-valued. Moreover, the optimal format to feed the real and imaginary part of the data to the NN is a cumbersome task. Fortunately, there exists an equivalent real-valued representation of the complexvalued MIMO system in (1) given by [8]

$$
\begin{array}{r}
\dot{\boldsymbol{x}}=\left[\mathscr{R}(\boldsymbol{x})^{\top} \mathscr{I}(\boldsymbol{x})^{\top}\right]^{\top} \\
\dot{\boldsymbol{y}}=\left[\mathscr{R}(\boldsymbol{y})^{\top} \mathscr{I}(\boldsymbol{y})^{\top}\right]^{\top} \\
\dot{\boldsymbol{n}}=\left[\mathscr{R}(\boldsymbol{n})^{\top} \mathscr{I}(\boldsymbol{n})^{\top}\right]^{\top} \\
\dot{\boldsymbol{H}}=\left[\begin{array}{cc}
\mathscr{R}(\boldsymbol{H}) & \mathscr{I}(\boldsymbol{H}) \\
-\mathscr{I}(\boldsymbol{H}) & \mathscr{R}(\boldsymbol{H})
\end{array}\right]
\end{array}
$$

where, $\dot{\boldsymbol{x}}$ is a $2 \times U$-dimensional real vector, $\dot{\boldsymbol{y}}$ and $\dot{\boldsymbol{n}}$ are $2 \times N$-dimensional real vectors, and $\dot{\boldsymbol{H}}$ is a $N \times(2 \times U)$ real matrix, capturing the real and imaginary parts of $\boldsymbol{x}$, $\boldsymbol{y}, \boldsymbol{n}$, and $\boldsymbol{H}$, respectively.

For the training phase, we map the transmitted symbols to vectors of ones and zeros using the one-hot encoding scheme. For example, consider a $2 \times 2$ MIMO 
with transmitted 16-QAM symbols being $s_{1}=3+1 i$, $s_{2}=3-1 i$. First, we convert the complex-valued vector to a real vector using (11), specifically, $\dot{\boldsymbol{s}}=[3,3,1,-1]^{\top}$. Converting this vector using one-hot encoding scheme maps it to a vector consisting only of ones and zeros, which is more suitable for training purposes. For example, considering 16-QAM, one-hot maper can be described as

$$
\begin{aligned}
& \operatorname{sym}_{1}=-3 \leftrightarrow[1,0,0,0] \\
& \operatorname{sym}_{2}=+3 \leftrightarrow[0,0,0,1] \\
& \operatorname{sym}_{3}=-1 \leftrightarrow[0,1,0,0] \\
& \operatorname{sym}_{4}=+1 \leftrightarrow[0,0,1,0] .
\end{aligned}
$$

Hence, the real and imaginary values of each symbol for 16-QAM are mapped to a row vector of four elements. For example, with $\dot{\boldsymbol{s}}=[3,3,1,-1]^{\top}$, through concatenation, $\dot{\boldsymbol{y}}_{\text {target }}=[1,0,0,0,1,0,0,0,0,0,1,0,0,1,0,0]^{\top}$. As for the input (feature) vector, consider the following transmit signal vector and channel matrix

$$
\begin{gathered}
\boldsymbol{x}=\left[\begin{array}{ll}
1.0036+0.7480 i & 6.3991-j 1.2315 i
\end{array}\right]^{\top} \\
\boldsymbol{H}=\left[\begin{array}{ll}
0.7378+0.5802 i & 0.0065-0.5814 i \\
0.2519+0.9350 i & 1.2762+0.8626 i
\end{array}\right] .
\end{gathered}
$$

After forming the estimation using equation (8), $\dot{x}_{\text {est }}=$ $\left[\begin{array}{llll}1.0036 & 6.3991 & 0.7480 & 1.2315\end{array}\right]^{\top}$. Hence, $\dot{x}_{\text {est }}$ is one sample (one row) of the the input matrix of the NN and $\dot{y}_{\text {target }}$ is the corresponding row in the output matrix of the NN. Looking at $\dot{y}_{\text {target }}$, one might think that this input feature is not sufficient for the classification task, however, it is imperative to realize that the NN is trained in a way that each output node corresponds to a real or an imaginary part of a label. Furthermore, for each output node, an independent classifier is trained based on the binary relevance method. For this example, $\dot{\boldsymbol{x}}_{\mathrm{est}}$ is used to predict real and imaginary parts of $s_{1}$ and $s_{2}$ using not only one but four different independent classifiers embedded within the same NN.

We train our model for $\mathbb{T}$ training symbols and channel variations. Therefore, $\dot{\boldsymbol{x}}_{\mathrm{est}}$ is a $\mathbb{T} \times(2 \times U)$ matrix, which is the input matrix of the NN and, $\dot{y}_{\text {target }}$, the $\mathbb{T} \times(8 \times U)$ target/label matrix, is the output matrix of the NN for training. We train the network with $80 \%$ of the samples and validate the model on the remaining $20 \%$. The trained model is then used to detect the symbols and calculate SER on a different test set.

\section{Simulation Results}

In this section, we investigate the SER performance of the proposed deep learning-based detection method, and accordingly draw comparisons against the conventional linear threshold-based method. Specifically, we carry out simulations for $2 \times 2$, and $4 \times 4$ multi-user MIMO systems, under BPSK and 16-QAM modulations, and for varying SNR values. Our training set for the NN consists of $10^{6}$ samples, and the test set has $10^{3}$ samples.

To design the NN, we use the keras deep learning library [10] . Further, we use the "LeakyReLU" activation function to introduce non-linearity properties to the network; the LeakyReLU allows a small positive gradient when the relevant neuron is not active, therefore tackles the well-known vanishing gradient problem. The output layer is a densely connected layer with the Sigmoid activation function. As previously highlighted, we convert the targets/labels vector to a vector of containing only ones and zeros, and for each target label an independent classifier is trained within the same NN. Thus, the model uses the binary cross-entropy function to calculate the loss. The optimization problem to minimize the respective loss is solved using the Adam optimizer [11], a variant of stochastic gradient descent algorithm. The number of the input/output neurons is defined with respect to the number of UEs and BS receive antennas. We utilize Grid-Search to find the number of hidden layers, and hidden neurons in each layer. We trained the network on a batch size of 32 and with $10^{3}$ epochs.

Fig. 2 illustrates the simulation results for the $2 \times 2$ MIMO system with different BPSK and 16-QAM modulations. We observe that the proposed detection based on NNs classifiers improves SER performance compared to detection under linear thresholds. For a target error rate performance, the proposed model, has achieved up to a $2 \mathrm{~dB}$ gain in SER. Moreover, in low SNR region, the gap between the performance of the two models becomes greater, because as the noise power increases, the classifier is better equipped to strip the estimated symbol of error compared to the conventional model. Under high SNR values, the two methods converge; this is expected, as with insignificant noise powers, the error term in the estimated signal is indeed small. It is worth mentioning that by improving the NN classifier, the performance gap between the two models may be larger.

Fig. 3 demonstrates the simulation results for the $4 \times 4$ MIMO system under BPSK and 16-QAM transmission schemes. Comparing Figs. 2 and 3, we observe, that as the MIMO dimension increases, the performance gap between the two detectors increases accordingly. On the other hand, if the number of users and receiver antennas in the BS for two systems are the same, the gap between the performance of the proposed model and the conventional model is higher for system with modulation of higher order. This shows as the modulation order increase, and we have a larger number of possible choices for the transmitted symbol, the NN-based method gain over the conventional method increases.

Overall, based on the above findings, it can be deduced that under a given transmit power budget, the 
$2 \times 2$ MIMO System

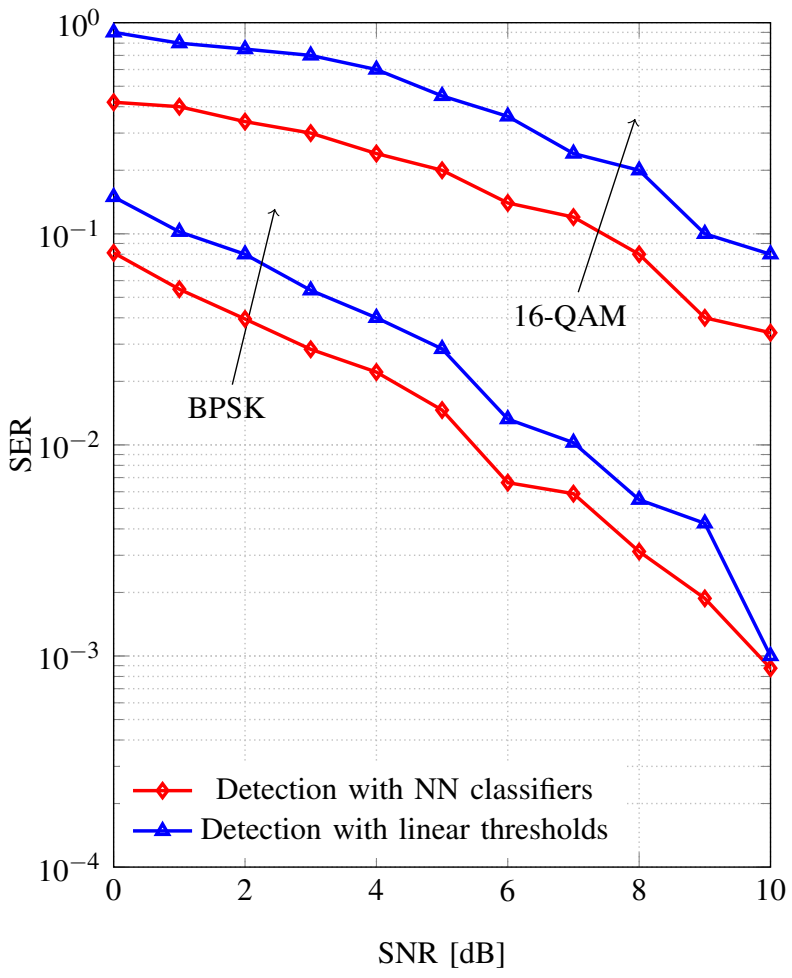

Fig. 2: SER performance of $2 \times 2$ MIMO under the proposed NN-based detector and conventional linear threshold model for different modulations and SNRs.

proposed NN-based detection model has achieved a lower SER versus the conventional method based on linear thresholds. Our results confirm that the use of deep learning tools for symbol decision boundary evaluation may be a powerful enabler for implementing high order modulations like 64-QAM and 256-QAM in a powerefficient manner.

\section{CONCLUSIONS}

In this work, we proposed a deep learning-based detection method for multi-user MIMO systems. We highlighted that the conventional detection methods are strictly sub-optimal in the context of MIMO fading channels as they resort to the use of linear thresholds. We developed a method in which the (near) optimal decision regions were formed by means of NN classifiers. Simulation results highlighted the promising error performance gain of the proposed method, which allows for achieving higher spectral efficiencies over the linear threshold-based detection methods. Future study can be done on further improvements to the NN architecture to achieve higher SER gains.

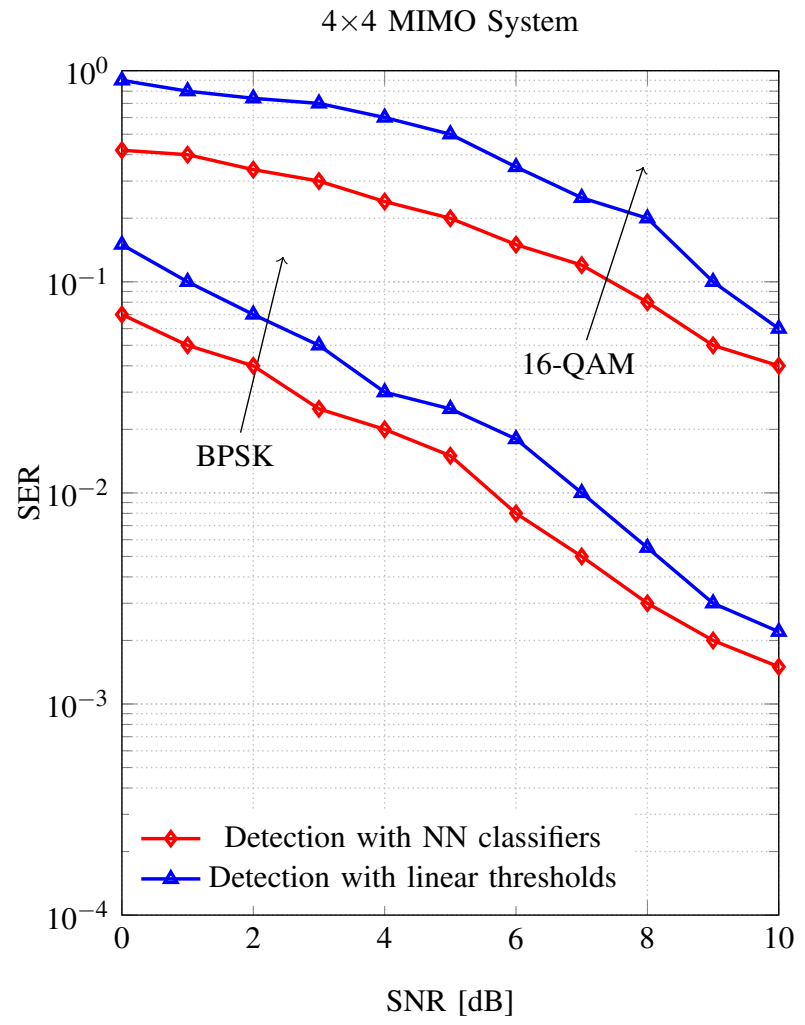

Fig. 3: SER performance of $4 \times 4$ MIMO under the proposed NN-based detector and conventional linear threshold model for different modulations and SNRs.

\section{REFERENCES}

[1] A. Zappone, M. Di Renzo, and M. Debbah, "Wireless networks design in the era of deep learning: Model-based, AI-based, or both?" arXiv:1902.02647, 2019.

[2] Z. Qin, H. Ye, G. Y. Li, and B.-H. F. Juang, "Deep learning in physical layer communications," arXiv:1807.11713, 2018.

[3] T. O'Shea and J. Hoydis, "An introduction to deep learning for the physical layer," IEEE Trans. Cogn. Commun. Netw., vol. 3, no. 4, pp. 563-575, 2017.

[4] T. J. O'Shea, T. Erpek, and T. C. Clancy, "Deep learning based MIMO communications," arXiv:1707.07980, 2017.

[5] N. Samuel, A. Wiesel, and T. Diskin, "Learning to detect," IEEE Trans. Signal Process., accepted 2019.

[6] S. Dorner, S. Cammerer, J. Hoydis, and S. ten Brink, "On deep learning-based communication over the air," in Asilomar Conf. Signals, Systems, Computers, Oct. 2017, pp. 1791-1795.

[7] J. Cioffi, "Signal processing and detection."

[8] T. Kailath, H. Vikalo, and B. Hassibi, "MIMO receive algorithms," Space-Time Wireless Systems: From Array Processing to MIMO Communications, 2005.

[9] O. Luaces, J. Díez, J. Barranquero, J. J. del Coz, and A. Bahamonde, "Binary relevance efficacy for multilabel classification," Progress in Artificial Intelligence, vol. 1, no. 4, pp. 303-313, Dec 2012.

[10] F. Chollet et al., "Keras," https://keras.io, 2015.

[11] D. P. Kingma and J. Ba, "Adam: A method for stochastic optimization," 2014. 